\title{
Use of antidepressant medications in relation to the incidence of breast cancer
}

\author{
D Fulton-Kehoe ${ }^{*, 1,2}$, MA Rossing ${ }^{2,3}$, C Rutter ${ }^{4}$, MT Mandelson ${ }^{3,4}$ and NS Weiss ${ }^{2,3}$ \\ 'Department of Environmental and Occupational Health Sciences, University of Washington, School of Public Health and Community Medicine, Seattle, \\ WA, USA; ${ }^{2}$ Department of Epidemiology, University of Washington, School of Public Health and Community Medicine, Seattle, WA, USA; ${ }^{3}$ Division of \\ Public Health Sciences, Fred Hutchinson Cancer Research Center, Seattle, WA, USA; ${ }^{4}$ Center for Health Studies, Group Health Cooperative, Seattle, WA, \\ USA
}

Although associations have been reported between antidepressant use and risk of breast cancer, the findings have been inconsistent. We conducted a population-based case-control study among women enrolled in Group Health Cooperative (GHC), a health maintenance organization in Washington State. Women with a first primary breast cancer diagnosed between 1990 and 200 I were identified $(N=2904)$ and five controls were selected for each case $(N=14396)$. Information on antidepressant use was ascertained through the GHC pharmacy database and on breast cancer risk factors and screening mammograms from $\mathrm{GHC}$ records. Prior to one year before diagnosis of breast cancer, about $20 \%$ of cases and controls had used tricyclic antidepressants (adjusted odds ratio $=1.06$, $95 \% \mathrm{Cl} 0.94-1.19)$ and $6 \%$ of each group had used selective serotonin reuptake inhibitors $(\mathrm{OR}=0.98,95 \% \mathrm{Cl} 0.80-1.18)$. There also were no differences between cases and controls with regard to the number of prescriptions filled or the timing of use. Taken as a whole, the results from this and other studies to date do not indicate an altered risk of breast cancer associated with the use of antidepressants overall, by class, or for individual antidepressants.

British Journal of Cancer (2006) 94, 107I-1078. doi:I0.1038/sj.bjc.66030 I7 www.bjcancer.com

Published online 7 March 2006

(C) 2006 Cancer Research UK

Keywords: breast cancer; antidepressant agents; case-control studies

Antidepressant use has increased steadily (Stafford et al, 2001; Pirraglia et al, 2003; Meijer et al, 2004) since the introduction of selective serotonin reuptake inhibitors (SSRIs) in the late 1980s, with use reported by $10-20 \%$ of women in recent studies (Moorman et al, 2003; Steingart et al, 2003; Chien et al, 2005), though actual use is likely to be higher due to underreporting (Boudreau et al, 2004).

Antidepressants may affect the risk of breast cancer through the influence of prolactin, of which elevated levels have been observed with use of SSRIs, tricyclic antidepressants, and other psychotropic drugs (Amsterdam et al, 1997; Cowen and Sargent, 1997; Emiliano and Fudge, 2004). Higher prolactin levels have been associated with an increased risk of postmenopausal breast cancer (Hankinson et al, 1999; Tworoger et al, 2004). However, the results of several studies of a possible association between antidepressant use and breast cancer risk have been inconsistent (see Discussion section).

To assess more completely the relation of breast cancer with antidepressant use, we conducted a large case control study using both computerized pharmacy records as well as records of reproductive history, family history of breast cancer, and other breast cancer risk factors. Breast cancer cases diagnosed during

*Correspondence: Dr D Fulton-Kehoe, Department of Environmental and Occupational Health Sciences, University of Washington, School of Public Health and Community Medicine, Seattle, WA, USA;

E-mail: debfk@u.washington.edu

Received 2 December 2005; revised 27 January 2006; accepted 27 January 2006; published online 7 March 2006
1990-2001 were included, which allowed examination of newer SSRIs as well as tricyclic antidepressants.

\section{METHODS}

All study subjects were enrollees of Group Health Cooperative (GHC), a health maintenance organization with over 500000 members in western Washington State. Women with a new diagnosis of invasive or in situ breast cancer diagnosed between 1990 and 2001 were identified by routine linkage between the GHC enrollment file and the Seattle-Puget Sound Surveillance, Epidemology, and End-Results SEER (2005). Five controls were selected for each case matched on age, calendar year, and length of GHC membership, with the diagnosis date of the case assigned to each matched control as the reference date. To ensure more complete pharmacy records, we restricted cases and controls to women continuously enrolled at GHC for at least 4 years. We identified 2904 eligible cases age 30-79 when diagnosed with a first primary invasive or in situ breast cancer and 14396 eligible controls.

Since 1977, the GHC pharmacy database has included a record for all prescriptions dispensed to GHC enrollees. Each pharmacy record includes a patient identifier, the drug type and dose, date dispensed, quantity dispensed, and dosing instructions. Measures of antidepressant exposure included all prescriptions from the GHC enrollment start date up to 1 year prior to the reference date, with the exception of time since last use, which also included prescriptions filled in the year before the reference date. To 
provide some assurance that a prescribed drug was actually taken, we classified women as antidepressant users if they filled at least two prescriptions in a 6-month interval. We used number of prescriptions filled as a measure of duration of use, with a typical prescription filled for 1 month.

We examined antidepressant use overall, individual antidepressant medications, and four classes of antidepressants including SSRIs, tricyclics, monoamine oxidase inhibitors (MAOIs), and atypical antidepressants. The SSRI category included paroxetine, fluoxetine, sertraline, fluvoxamine, and citalopram. Tricyclic antidepressants included amitriptyline, doxepin, imipramine, desipramine, nortriptyline, protriptyline, amoxapine, trimipramine, and clomipramine. The MAOIs included phenelzine and tranylcypromine. Bupropion, mirtazapine, nefazodone, venlafaxine, maprotiline (a tetracyclic antidepressant), and trazodone were classified as 'atypical' antidepressants.

Information on breast cancer risk factors and mammography history were obtained from the GHC Breast Cancer Screening Program (BCSP) databases (Taplin et al, 1990). As part of the Breast Cancer Screening Program, self-administered questionnaires are first sent to all GHC women when they turn 40 (or enroll in GHC, whichever is later). Of eligible women, approximately $85-90 \%$ enroll in the BCSP and complete the initial questionnaire. The questionnaire includes information on clinical breast exams, previous mammograms, menopausal status, use of menopausal hormone replacement therapy, age at first birth, previous biopsies, family history of breast cancer, height, weight, education, and race. Selected questions from the BCSP questionnaire are updated at each screening visit. GHC also maintains databases of mammograms performed at GHC with dates of each mammogram, indications for examination (screening or diagnostic), type of evaluation (standard screening or diagnostic), results, and recommendations. Based on these records, we determined whether a study participant had had a screening mammogram in the 2 years prior to her diagnosis or reference date.

Unconditional logistic regression was used to compute odds ratios (as an estimate of relative risk) and 95\% CI for duration and type of antidepressant use in relation to risk of breast cancer. All models included the matching factors (age at reference date, length of GHC membership, and reference year) as continuous variables. Family history of breast cancer, use and duration of HRT, body mass index, parity/age at first birth, and mammographic screening in the 2 years before reference date were determined to be potential confounders based on their associations with both exposure and disease. In addition to the matching factors, these factors were included in all multivariate models.

Our primary analyses included all women with invasive breast cancer and controls. Additional analyses examined ductal and lobular cases separately. We also conducted analyses separately of women with oestrogen receptor (ER) positive and ER negative tumours, based on routinely collected information from SEER. Polytomous regression models were used when cases were classified based on histology or ER status, which resulted in multiple case groups. To ensure more complete exposure data, some analyses were restricted to the subgroup of women enrolled in GHC for at least 10 years (over $70 \%$ of eligible subjects in this study). Cases with in situ disease were examined separately due to differences in the natural history (Bodian, 1993; Jensen and Page, 2003) and in some risk factors (Weiss et al, 1996; Trentham-Dietz et al, 2000; Moorman et al, 2003) between invasive and in situ disease.

\section{RESULTS}

Of the 2904 eligible cases, 2449 had invasive breast cancer and 455 had in situ carcinoma. Of the cases with invasive disease, $72 \%$ were ductal, $14 \%$ lobular or mixed lobular and ductal, and $14 \%$ were other histologies. Compared to controls, women with invasive breast cancer were more likely to report a family history of breast cancer, to be nulliparous, and to be at least 30 years old at first birth (Table 1). A higher percent of cases reported ever use of HRT and longer use of HRT. Women with invasive disease were slightly more likely to be overweight or obese than controls. The percentage of subjects with a screening mammogram within 2 years of the reference date was higher for invasive cases (71\%) than for controls (58\%) and cases were more likely to report a previous breast biopsy than controls.

Overall, $34 \%$ of women had filled at least 1 prescription for antidepressants up to 1 year before the diagnosis or reference date. About $24 \%$ had filled at least 2 antidepressant prescriptions within 6 months and were considered antidepressant users for this study. About $20 \%$ had filled at least 2 prescriptions for tricyclic antidepressants. For SSRIs, $6 \%$ had filled at least two prescriptions within 6 months and about $6 \%$ filled at least two prescriptions for atypical antidepressants. For each antidepressant class, women filled a median of 10 prescriptions.

Among controls, women who used antidepressants were more likely to report use of HRT than nonusers and had a longer duration of use than women who had not used antidepressants (Table 2). A higher percentage of women who used antidepressants were classified as obese than those who did not use antidepressants. Antidepressant users were also more likely to have had a screening mammogram within 2 years before the reference date and had been enrolled at GHC for a longer time than nonusers. Women who were older, had a younger age at menarche, a first birth before 20 years of age, or were white were more likely to use antidepressants, although these differences were relatively small.

When only adjusted for matching factors, the odds ratio for ever use of antidepressants was slightly elevated (OR $=1.1195 \% \mathrm{CI}$ $1.00-1.22, P=0.048$ ) (Table 3). After adjusting for potential confounding variables the OR was $1.04(95 \% \mathrm{CI}=0.94-1.16)$. Risk did not increase with increasing number of antidepressant prescriptions filled. The odds ratio for tricyclic use was slightly elevated when adjusted only for matching factors $(\mathrm{OR}=1.12,95 \%$ CI $1.00-1.24)$, but not after additional adjustment $(\mathrm{OR}=1.06,95 \%$ CI 0.94-1.19). The risk was not elevated with a greater number of tricyclic prescriptions filled. Ever use of SSRIs also was not associated with breast cancer risk (adjusted OR $=0.98,95 \% \mathrm{CI}$ $0.80-1.18)$, nor was receipt of a relatively larger number of prescriptions. Use of atypical antidepressants was not associated with an increased risk of breast cancer.

In the analyses of the three most common tricyclic medications (Table 4), there was no association with doxepin or imipramine, but the odds ratios were somewhat elevated for amitriptyline. When accounting only for the matching factors, the odds ratio for ever use of amitriptyline was 1.27 (95\% CI $1.10-1.47$ ). The odds ratio was somewhat attenuated, but still slightly elevated $(\mathrm{OR}=1.21,95 \%$ CI $1.03-1.41)$ after adjusting for potential confounding factors. No elevated risks were observed for the three most common SSRIs (fluoxetine, paroxetine, or sertraline) or with trazodone, the most common atypical antidepressant.

No patterns of increased risk were seen with time since first use or time since last use of tricyclics or SSRIs (data not shown).

In analyses by ER status, no associations were seen between antidepressant use overall, number of antidepressant prescriptions, SSRI use, number of SSRI prescriptions, tricyclic use, or number of tricyclic prescriptions with either ER + or ER- breast cancer (Table 5). With the possible exception of amitriptyline, individual antidepressants were not associated with either ER + or ER - breast cancer. In fully adjusted models for ER + breast cancer, the OR for ever use of amitriptyline was 1.22 (95\% CI 1.021.45). For 2-10 prescriptions of amitriptyline the OR was 1.19 (95\% CI $0.95-1.48)$ and for 11 or more prescriptions the OR was 1.27 (95\% CI $0.96-1.66)$. No associations were observed with other individual antidepressant medications (data not shown). 
Antidepressants and breast cancer

Table I Characteristics of women with invasive breast cancer and controls

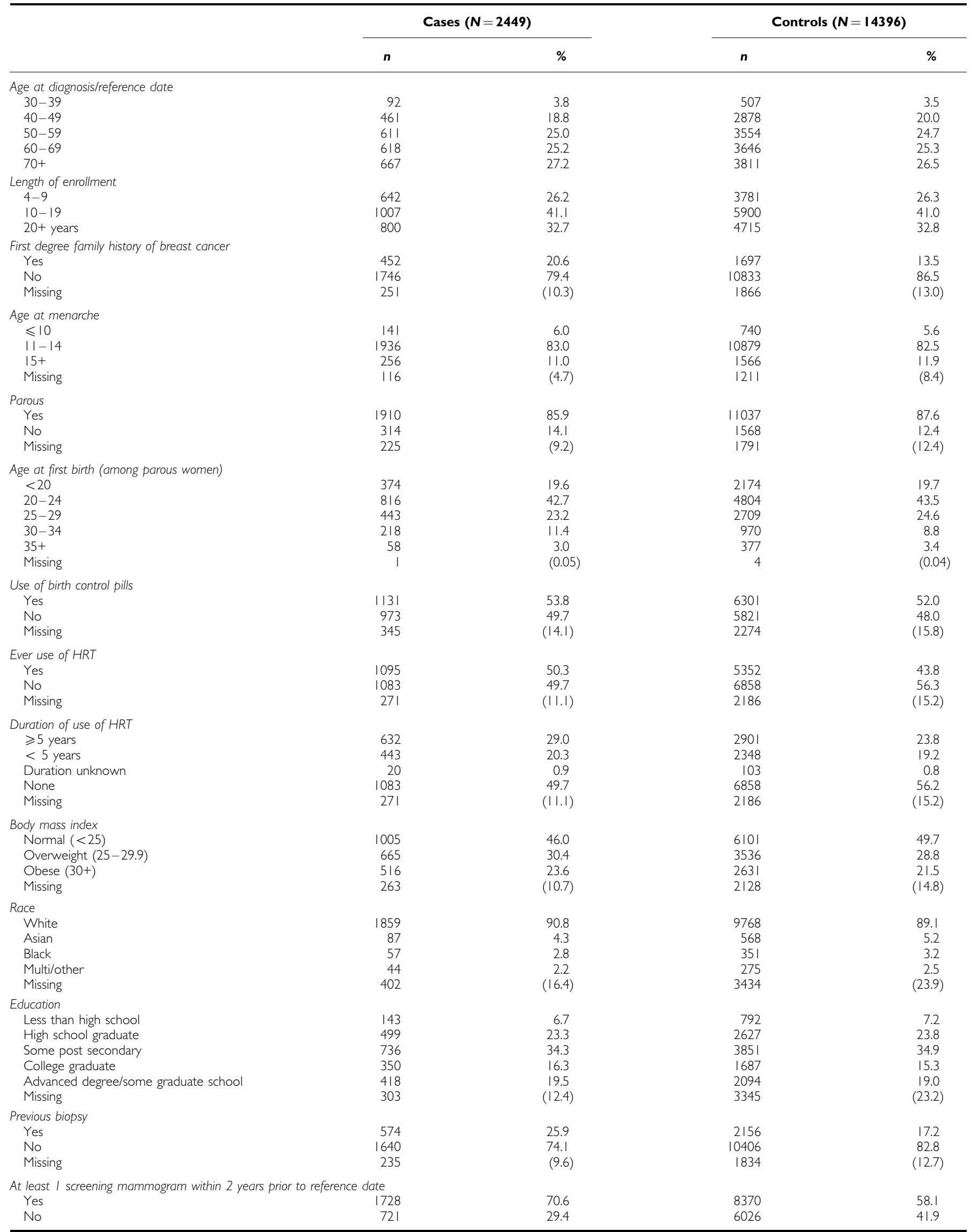


Table 2 Characteristics of antidepressant users and nonusers among controls

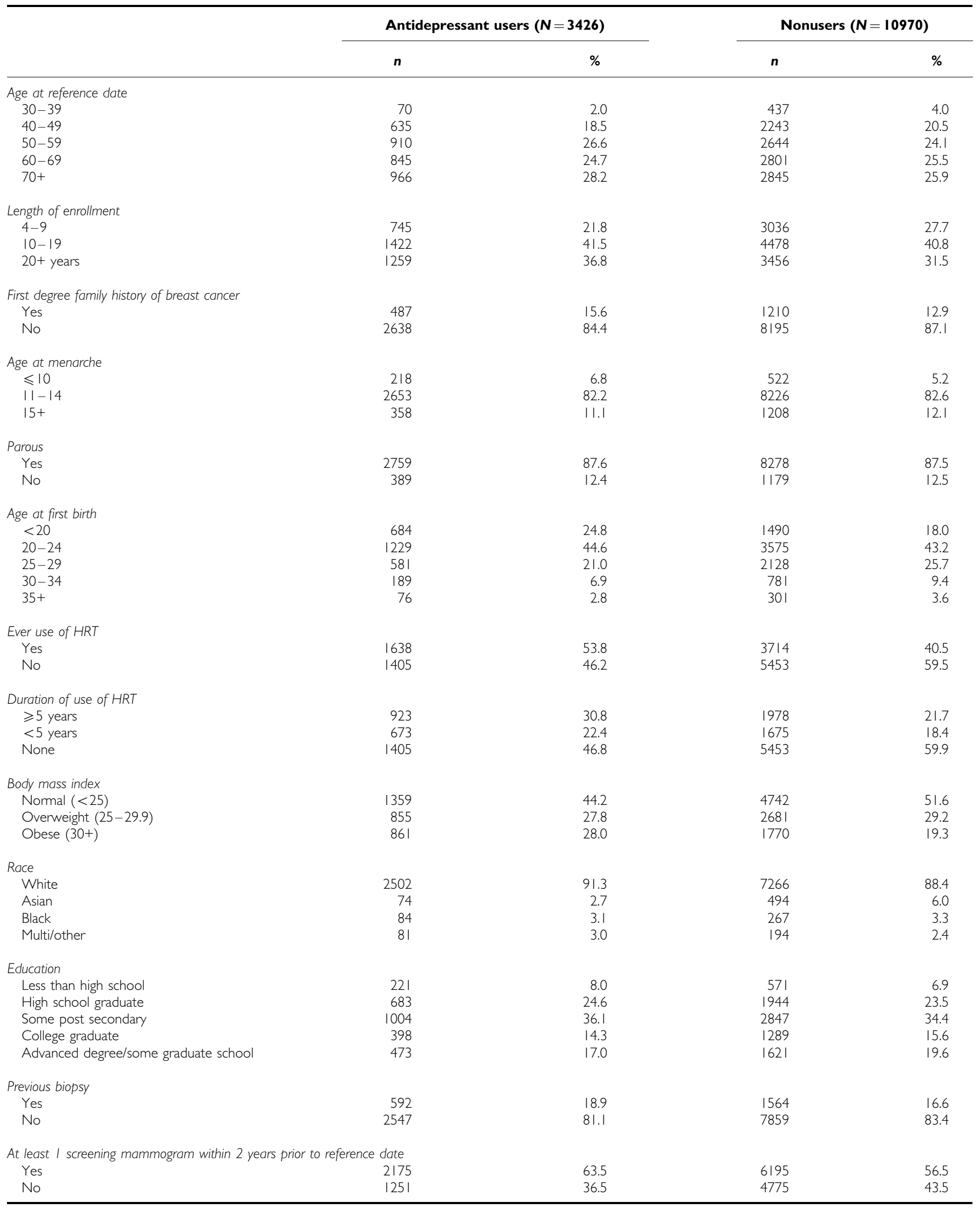


Table 3 Antidepressant use among women with invasive breast cancer and controls

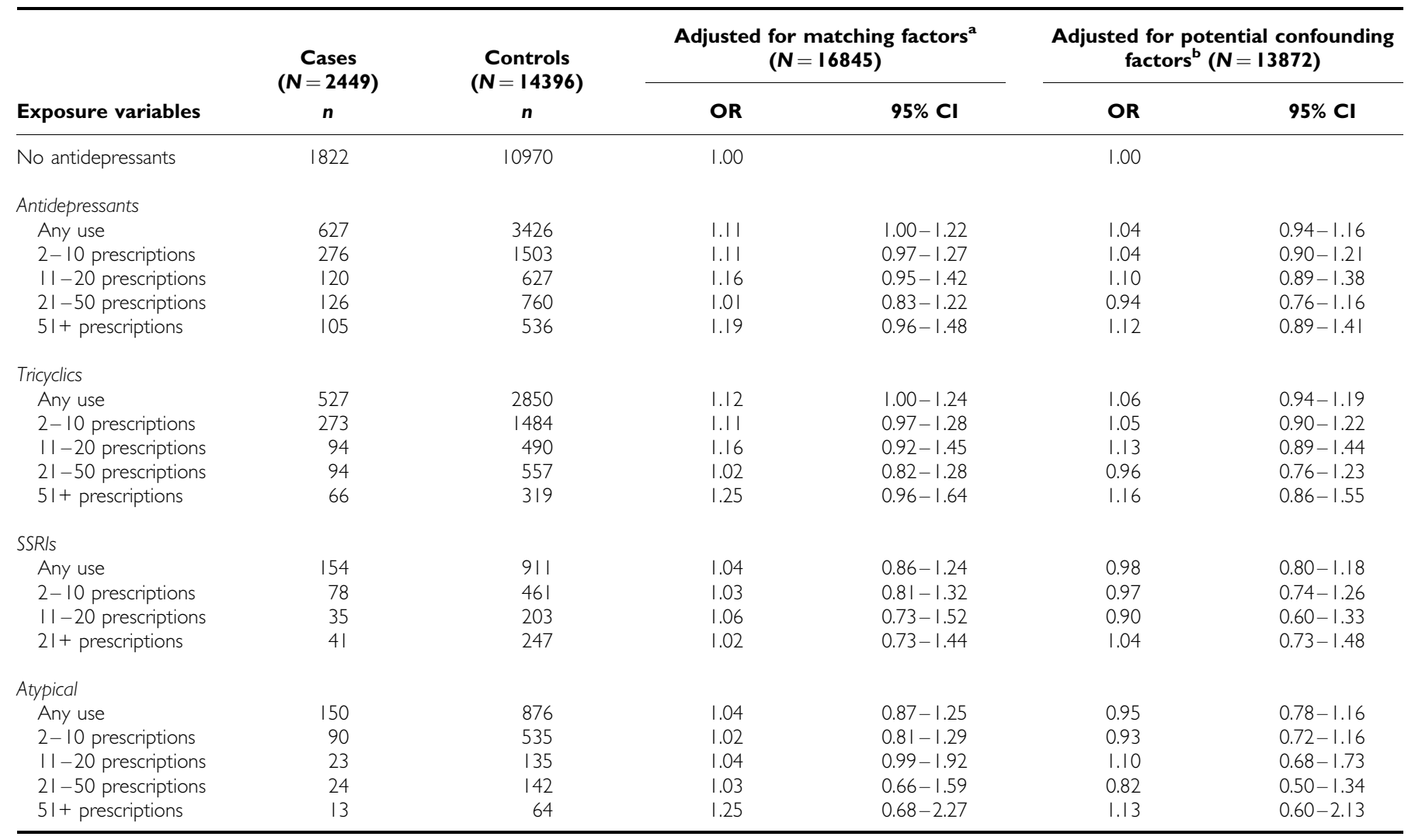

${ }^{a}$ Adjusted for age, length of enrollment, and calendar year. ${ }^{b}$ Adjusted for age, length of enrollment, calendar year, family history of breast cancer, parity/age at first birth, duration of HRT use, body mass index, and history of screening mammogram in 2 years prior to reference date.

In the analysis of cases with invasive ductal carcinoma (data not shown), the odds ratios for the highest category of antidepressant use $(\mathrm{OR}=1.31,95 \% \mathrm{CI} 1.02-1.68)$ and the highest category of tricyclic use $(\mathrm{OR}=1.31,95 \% \mathrm{CI} 1.00-1.88)$ were somewhat elevated after adjusting for potential confounders. Risk was not elevated in any of the other categories of use, with no indication of a dose-response trend. No associations were seen between risk of ductal carcinoma and use of SSRIs or atypical antidepressants. Antidepressant use was not associated with invasive lobular carcinoma.

When analyses were restricted to women enrolled in GHC for at least 10 years, risks did not differ for antidepressants overall or any class of antidepressants (data not shown). Neither any use of antidepressants overall nor use of a particular class of antidepressants was associated with an increased risk of carcinoma in situ (data not shown).

\section{DISCUSSION}

With few exceptions, no increased risks were observed between antidepressant use and risk of invasive breast cancer. After adjustment for potential confounding factors no associations were seen between risk of breast cancer and antidepressants overall, SSRIs, tricyclics, or atypical antidepressants. No increased risk was observed with time since first or last use of any class of antidepressants. When individual antidepressants were examined, only amitriptyline exhibited a possible, albeit weak, association $(\mathrm{OR}=1.21,95 \% \mathrm{CI} 1.03-1.41)$. Risks were not elevated for any other individual medications.

Similar to our study, most prior studies have found little or no association with ever use of antidepressants (all classes combined)
(Cotterchio et al, 2000; Wang et al, 2001; Moorman et al, 2003; Steingart et al, 2003; Chien et al, 2005), with increasing duration of use (Cotterchio et al, 2000; Wang et al, 2001; Moorman et al, 2003; Steingart et al, 2003; Chien et al, 2005), or with time since first or last use (Steingart et al, 2003; Chien et al, 2005).

For use of tricyclic antidepressants, no increased risks were seen in most studies for current (Chien et al, 2005; Gonzalez-Perez and Garcia Rodriguez, 2005) or ever use (Kelly et al, 1999; Cotterchio et al, 2000; Wang et al, 2001; Sharpe et al, 2002; Moorman et al, 2003; Steingart et al, 2003; Chien et al, 2005). In the present study, there was a weak positive association when adjusted only for matching factors and this was attenuated when adjusted for additional potential confounders. Longer duration of tricyclic use was associated with an increased risk of breast cancer $(\mathrm{OR}=2.1$, 95\% CI 0.9-5.0) in one study (Cotterchio et al, 2000), but not in others (Moorman et al, 2003; Gonzalez-Perez and Garcia Rodriguez, 2005), including the present study, although cutpoints for the highest category of use have varied. Although we found a slightly increased risk with use of amitriptyline, other studies that examined individual tricyclic medications observed no increased risks associated with use of this drug (Kelly et al, 1999; Cotterchio et al, 2000; Wang et al, 2001; Steingart et al, 2003). Sharpe et al (2002) reported an elevated risk of breast cancer associated with the tricyclic drugs classified as 'genotoxic' based on Drosophila wing development (van Schaik and Graf, 1991; van Schaik and Graf, 1993). In the present study, no increased risk was observed either with an increasing number of prescriptions or timing of use of these same potentially genotoxic tricyclic medications (data not shown). In fact, in contrast to the findings by Sharpe et al, we found a slightly elevated risk of breast cancer associated with use of nongenotoxic tricyclic drugs, which includes the drug amitriptyline. 
Table 4 Use of specific antidepressant medications among women with invasive breast cancer and controls

\begin{tabular}{|c|c|c|c|c|c|c|}
\hline \multirow[b]{2}{*}{ Exposure variable } & \multirow{2}{*}{$\begin{array}{c}\text { Cases } \\
n\end{array}$} & \multirow{2}{*}{$\begin{array}{c}\text { Controls } \\
n\end{array}$} & \multicolumn{2}{|c|}{ Adjusted for matching factors ${ }^{a}$} & \multicolumn{2}{|c|}{ Adjusted for potential confounding factors ${ }^{b}$} \\
\hline & & & OR & $95 \% \mathrm{Cl}$ & OR & $95 \% \mathrm{Cl}$ \\
\hline No antidepressants & 1822 & 10970 & 1.00 & & 1.00 & \\
\hline \multicolumn{7}{|l|}{ Tricyclics } \\
\hline \multicolumn{7}{|l|}{ Amitriptyline } \\
\hline Any use & 250 & |19| & 1.27 & $1.10-1.47$ & 1.21 & $1.03-1.4 \mid$ \\
\hline $2-10$ prescriptions & $15 \mid$ & 756 & 1.20 & $1.00-1.44$ & 1.17 & $0.97-1.43$ \\
\hline $11+$ prescriptions & 99 & 435 & 1.38 & $1.10-1.72$ & 1.26 & $0.99-1.60$ \\
\hline \multicolumn{7}{|l|}{ Doxepin } \\
\hline Any use & 186 & $110 \mid$ & 1.01 & $0.86-1.19$ & 0.95 & $0.79-1.13$ \\
\hline $2-10$ prescriptions & 120 & 710 & 1.01 & $0.83-1.23$ & 0.94 & $0.75-1.17$ \\
\hline II+ prescriptions & 66 & 391 & 1.01 & $0.78-1.32$ & 0.96 & $0.72-1.27$ \\
\hline \multicolumn{7}{|l|}{ Imipramine } \\
\hline Any use & 127 & 683 & 1.13 & $0.93-1.37$ & 1.04 & $0.84-1.29$ \\
\hline $2-10$ prescriptions & 79 & 444 & 1.08 & $0.84-1.38$ & 0.98 & $0.76-1.28$ \\
\hline II+ prescriptions & 48 & 239 & 1.22 & $0.89-1.67$ & 1.15 & $0.82-\mid .61$ \\
\hline \multicolumn{7}{|l|}{$S S R / S$} \\
\hline \multicolumn{7}{|l|}{ Fluoxetine } \\
\hline Any use & 114 & 611 & 1.07 & $0.86-1.34$ & 1.00 & $0.80-1.25$ \\
\hline $2-10$ prescriptions & 59 & 364 & 0.99 & $0.75-1.31$ & 0.94 & $0.69-1.27$ \\
\hline II+ prescriptions & 55 & 297 & 1.14 & $0.85-1.53$ & 1.07 & $0.78-1.46$ \\
\hline \multicolumn{7}{|l|}{ Paroxetine } \\
\hline Any use & 41 & 241 & 1.05 & $0.75-1.48$ & 1.00 & $0.70-1.41$ \\
\hline $2-10$ prescriptions & 36 & 178 & 1.25 & $0.87-|.8|$ & 1.15 & $0.79-1.69$ \\
\hline II+ prescriptions & 5 & 63 & 0.49 & $0.20-1.23$ & 0.52 & $0.21-|.3|$ \\
\hline \multicolumn{7}{|l|}{ Sertraline } \\
\hline Any use & 40 & 215 & 1.15 & $0.82-1.62$ & 1.16 & $0.81-1.66$ \\
\hline $2-10$ prescriptions & 24 & 129 & 1.15 & $0.74-1.78$ & 1.18 & $0.75-1.86$ \\
\hline II+ prescriptions & 16 & 86 & 1.15 & $0.67-1.98$ & 1.12 & $0.64-1.98$ \\
\hline \multicolumn{7}{|l|}{ Atypical } \\
\hline \multicolumn{7}{|l|}{ Trazodone } \\
\hline Any use & $|4|$ & 802 & 1.07 & $0.89-1.29$ & 0.96 & $0.78-1.18$ \\
\hline $2-10$ prescriptions & 82 & 502 & 0.99 & $0.78-1.26$ & 0.88 & $0.68-1.15$ \\
\hline I I+ prescriptions & 59 & 300 & 1.20 & $0.90-1.59$ & 1.09 & $0.80-1.49$ \\
\hline
\end{tabular}

${ }^{a}$ Adjusted for age, length of enrollment, and calendar year. ${ }^{b}$ Adjusted for age, length of enrollment, calendar year, family history of breast cancer, parity/age at first birth, duration of HRT use, body mass index, history of screening mammogram in 2 years prior to reference date.

Most studies (Kelly et al, 1999; Cotterchio et al, 2000; Wang et al, 2001; Moorman et al, 2003; Coogan et al, 2005; GonzalezPerez and Garcia Rodriguez, 2005) have not found an association between ever use of SSRIs and risk of breast cancer, but one reported a slight increased risk $(\mathrm{OR}=1.32,95 \%$ CI $0.97-1.80)$ (Steingart et al, 2003). Although recent use of SSRIs was associated with an increased risk of breast cancer in one study (Kelly et al, 1999), our study and others (Chien et al, 2005; Gonzalez-Perez and Garcia Rodriguez, 2005) that examined timing of use did not find a similar association. Duration of SSRI use has been assessed in this and other studies (Kelly et al, 1999; Cotterchio et al, 2000; Moorman et al, 2003; Chien et al, 2005; Coogan et al, 2005; Gonzalez-Perez and Garcia Rodriguez, 2005). One reported a doubling of the risk $(\mathrm{OR}=2.2,95 \% \mathrm{CI}=0.8-6.3)$ with duration of use longer than 3 years (Moorman et al, 2003), but others have not observed an increased risk with increasing duration of use, although few women had used SSRIs for more than 2-3 years.

Individual SSRI medications have been associated with an increased risk of breast cancer in two previous studies (Cotterchio et al, 2000; Steingart et al, 2003), but not in others (Chien et al, 2005; Coogan et al, 2005; Gonzalez-Perez and Garcia Rodriguez, 2005; Haque et al, 2005) including our current study. Although one study reported an elevated odds ratio associated with use of sertraline (OR $=1.45,95 \%$ CI $0.88-2.40)$ (Steingart et al, 2003), in our study and in others (Cotterchio et al, 2000; Coogan et al, 2005) the risk was not increased. The risk associated with paroxetine use has been substantially ( $\mathrm{OR}=7.2,95 \% \mathrm{CI} 0.9-58.3)$ (Cotterchio et al, 2000) and moderately $(\mathrm{OR}=1.45,95 \%$ CI $0.88-2.40)$ (Steingart et al, 2003) elevated in two studies, but not in ours or others (Chien et al, 2005; Coogan et al, 2005; Gonzalez-Perez and Garcia Rodriguez, 2005; Haque et al, 2005). We did not find an association with ever use of paroxetine (OR 1.00) or with increasing number of paroxetine prescriptions filled (OR 1.15 for 2-10 prescriptions and 0.52 for at least 11 prescriptions).

Atypical antidepressants have not shown an increased risk in this or other studies (Moorman et al, 2003; Chien et al, 2005). Trazodone was a commonly used antidepressant in our study population, but the number of women reporting use of atypical antidepressants has been quite low in other studies. Some studies relying on self-report may underestimate trazodone use if other indications of antidepressant use (such as insomnia) are not assessed.

The one other study that evaluated whether the risk associated with antidepressant use varies by ER status reported associations of antidepressant use with ER - tumors and with PR - tumors. The strongest association was observed with SSRIs in ER $+/ P R-$ cases 
Table 5 Antidepressant use among women with invasive breast cancer, by ER status, and controls

\begin{tabular}{|c|c|c|c|c|c|c|c|}
\hline \multirow[b]{2}{*}{ Exposure variables } & \multirow{2}{*}{$\begin{array}{c}\text { ER+ cases } \\
n\end{array}$} & \multirow{2}{*}{$\begin{array}{c}\text { ER- cases } \\
n\end{array}$} & \multirow{2}{*}{$\begin{array}{c}\text { Controls } \\
n\end{array}$} & \multicolumn{2}{|c|}{ ER+ compared to controls ${ }^{a}$} & \multicolumn{2}{|c|}{ ER- compared to controlsa } \\
\hline & & & & OR & $95 \% \mathrm{Cl}$ & OR & $95 \% \mathrm{Cl}$ \\
\hline \multicolumn{8}{|l|}{ Antidepressant } \\
\hline Any use & 457 & 106 & 3426 & 1.05 & $0.93-1.19$ & 1.01 & $0.79-1.30$ \\
\hline $2-10$ prescriptions & 207 & 45 & 1503 & 1.09 & $0.92-1.29$ & 0.93 & $0.65-1.33$ \\
\hline $51+$ prescriptions & 71 & 20 & 536 & 1.04 & $0.79-1.36$ & 1.39 & $0.86-2.26$ \\
\hline \multicolumn{8}{|l|}{ Tricyclic } \\
\hline Any use & 386 & 84 & 2850 & 1.07 & $0.94-1.21$ & 0.95 & $0.72-1.24$ \\
\hline $2-10$ prescriptions & 200 & 48 & 1484 & 0.97 & $0.68-1.38$ & 1.06 & $0.90-1.26$ \\
\hline II-20 prescriptions & 74 & 12 & 490 & 0.92 & $0.51-1.66$ & 1.19 & $0.92-1.55$ \\
\hline $21-50$ prescriptions & 67 & 10 & 557 & 0.55 & $0.27-1.13$ & 0.94 & $0.72-1.25$ \\
\hline $11-20$ prescriptions & 26 & 7 & 203 & 0.95 & $0.61-1.47$ & 0.87 & $0.35-2.15$ \\
\hline $21+$ prescriptions & 27 & 8 & 247 & 0.93 & $0.62-1.41$ & 1.21 & $0.58-2.51$ \\
\hline \multicolumn{8}{|l|}{ Atypical } \\
\hline Any use & 108 & 31 & 876 & 0.92 & $0.73-1.16$ & 1.15 & $0.76-1.74$ \\
\hline $2-10$ prescriptions & 66 & 19 & 535 & 0.93 & $0.70-1.23$ & 1.11 & $0.66-1.86$ \\
\hline II-20 prescriptions & 17 & 6 & 135 & 1.06 & $0.62-1.80$ & 1.76 & $0.76-4.07$ \\
\hline $21+$ prescriptions & 25 & 6 & 206 & 0.82 & $0.52-1.31$ & 0.89 & $0.36-2.20$ \\
\hline \multicolumn{8}{|l|}{ Amitriptyline } \\
\hline Any use & 186 & 38 & 1191 & 1.22 & $1.02-1.45$ & 1.09 & $0.75-1.58$ \\
\hline $2-10$ prescriptions & 113 & 23 & 756 & 1.19 & $0.95-1.48$ & 1.05 & $0.66-1.67$ \\
\hline $11+$ prescriptions & 73 & 15 & 435 & 1.27 & $0.96-1.66$ & 1.16 & $0.65-2.06$ \\
\hline
\end{tabular}

${ }^{a}$ Adjusted for age, length of enrollment, calendar year, family history of breast cancer, parity/age at first birth, duration of HRT use, body mass index, and history of screening mammogram in 2 years prior to reference date.

$(\mathrm{OR}=2.0,95 \%$ CI 1.1-3.8)(Chien et al, 2005). We did not find an association between SSRIs, tricyclics, or antidepressant use overall with either ER + or ER- breast cancers. In contrast to the earlier study (Chien et al, 2005), when we examined ER/PR combinations, risks were not elevated for $\mathrm{ER}+/ \mathrm{PR}-$ cases (data not shown).

Recently, one study reported that antidepressant users had a lower risk of breast cancer among women with a family history of breast cancer and a higher risk in those without a family history (Chien et al, 2005). Our results do not replicate this interaction (data not shown).

Much of the prior research examining antidepressants and breast cancer risk has relied on the self-reported use of antidepressants (Kelly et al, 1999; Cotterchio et al, 2000; Moorman et al, 2003; Steingart et al, 2003; Chien et al, 2005), but recall of prescription drugs varies according to the type of drug, the frequency of use, time since medication use, and education level (Saunders et al, 1994; West et al, 1995). Compared to pharmacy data, antidepressant use is substantially under-reported (Boudreau et al, 2004). A study comparing self-reported use of antihypertensives, statins, and antidepressants with pharmacy data found higher agreement for antihypertensives and statins than for antidepressant medications. Antidepressant use was recalled only $62 \%$ of the time within 6 months prior to reference date, compared to $94 \%$ for antihypertensive use (Boudreau et al, 2004). In addition, recall of names and dose of medication is relatively low (West et al, 1995) and the percentage missing timing of use is relatively high (Boudreau et al, 2004).

One strength of this study is the use of the GHC pharmacy records. Advantages of using administrative pharmacy records include having information available for all subjects (not only respondents), no under-reporting of use, no recall bias, and very complete information on drug name, dose, and timing of use. As most studies that use pharmacy databases to assess medication exposure have limited information on potential confounding factors (Wang et al, 2001; Sharpe et al, 2002; Gonzalez-Perez and Garcia Rodriguez, 2005; Haque et al, 2005), another important strength of the current study is the availability of breast cancer risk factor data.

One potential weakness of this study is the possibility that some women may have been prescribed antidepressants that were not included in the GHC pharmacy database. Prescriptions that were filled prior to the initiation of the pharmacy database in 1977, prior to enrollment in GHC, or filled out of the GHC system were not captured in the pharmacy databases. However, the number of antidepressants prescriptions filled outside the GHC system is likely to be small because antidepressant prescriptions were available free or with a small copayment for many GHC members. Further, others have reported that over $95 \%$ of prescriptions for antidepressants among GHC members are filled at a GHC pharmacy (Saunders et al, 1994). While we could not identify antidepressant use prior to GHC membership, we conducted subset analyses among women with at least 10 years of pharmacy data to ensure more complete history of antidepressant use. Over $70 \%$ of the subjects in this study were enrolled for more than 10 years as of their reference date and the conclusions based on this restricted sample were unchanged.

Another potential limitation of this study is a relatively short follow-up time for paroxetine exposure. Paroxetine was on the 
market in the United States in 1993, but because it was not a preferred drug in the GHC formulary, it was not used often within GHC until 1997. As a result, fewer subjects in this study population had ever used paroxetine and the duration of use was shorter than what would be expected in the general population during the same time period.

Overall, the results from this and other epidemiologic studies do not provide evidence for an increased risk of breast cancer associated with the use of antidepressants overall, by class of drug, or for individual antidepressants. Although a few studies have reported elevated risks in select analyses, the findings have not been consistent by class of drug, type of individual drug, or timing of use. The lack of consistency across studies suggests that any observed elevated risks could be due to chance. If there were a causal relationship, breast cancer risk would likely increase with longer duration of exposure. A few studies have reported elevated risks in longer-term users of some agents (Cotterchio et al, 2000; Moorman et al, 2003), but the class has differed. Overall, risk of breast cancer has not varied in any systematic pattern by timing of antidepressant use. As SSRIs have only been available in the relatively recent past, we cannot rule out the possibility of an effect with longer duration or longer time since first use, but the accumulating evidence does not suggest an association.

\section{ACKNOWLEDGEMENTS}

We would like to thank Robert Harrison from Group Health Cooperative for his assistance with compiling data for this project. This research was funded by the National Cancer Institute (Grant No. RO3 CA 94745).

\section{REFERENCES}

Amsterdam JD, Garcia-Espana F, Goodman D, Hooper M, Hornig-Rohan M (1997) Breast enlargement during chronic antidepressant therapy. J Affect Disord 46: $151-156$

Bodian CA (1993) Benign breast diseases, carcinoma in situ, and breast cancer risk. Epidemiol Rev 15: $177-187$

Boudreau DM, Daling JR, Malone KE, Gardner JS, Blough DK, Heckbert SR (2004) A validation study of patient interview data and pharmacy records for antihypertensive, statin, and antidepressant medication use among older women. Am J Epidemiol 159: 308-317

Chien C, Li CI, Heckbert SR, Malone KE, Boudreau DM, Daling JR (2005) Antidepressant use and breast cancer risk. Breast Cancer Res Treat 1-10

Coogan PF, Palmer JR, Strom BL, Rosenberg L (2005) Use of selective serotonin reuptake inhibitors and the risk of breast cancer. $A m J$ Epidemiol 152: $835-838$

Cotterchio M, Kreiger N, Darlington G, Steingart A (2000) Antidepressant medication use and breast cancer risk. Am J Epidemiol 151: 951 - 957

Cowen PJ, Sargent PA (1997) Changes in plasma prolactin during SSRI treatment: evidence for a delayed increase in 5-HT neurotransmission. J Psychopharmacol 11: $345-348$

Emiliano AB, Fudge JL (2004) From galactorrhea to osteopenia: rethinking serotonin-prolactin interactions. Neuropsychopharmacology 29: 833-846

Gonzalez-Perez A, Garcia Rodriguez LA (2005) Breast cancer risk among users of antidepressant medications. Epidemiology 16: 101-105

Hankinson SE, Willett WC, Michaud DS, Manson JE, Colditz GA, Longcope C, Rosner B, Speizer FE (1999) Plasma prolactin levels and subsequent risk of breast cancer in postmenopausal women. J Natl Cancer Inst 91: $629-634$

Haque R, Enger SM, Chen W, Petitti DB (2005) Breast cancer risk in a large cohort of female antidepressant medication users. Cancer Lett 221: $61-65$

Jensen RA, Page DL (2003) Ductal carcinoma in situ of the breast: impact of pathology on therapeutic decisions. Am J Surg Pathol 27: 828-831

Kelly JP, Rosenberg L, Palmer JR, Rao RS, Strom BL, Stolley PD, Zauber AG, Shapiro S (1999) Risk of breast cancer according to use of antidepressants, phenothiazines, and antihistamines. Am J Epidemiol 150: $861-868$

Meijer WE, Heerdink ER, Leufkens HG, Herings RM, Egberts AC, Nolen WA (2004) Incidence and determinants of long-term use of antidepressants. Eur J Clin Pharmacol 60: 57-61

Moorman PG, Grubber JM, Millikan RC, Newman B (2003) Antidepressant medications and their association with invasive breast cancer and carcinoma in situ of the breast. Epidemiology 14: 307-314

Pirraglia PA, Stafford RS, Singer DE (2003) Trends in prescribing of selective serotonin reuptake inhibitors and other newer antidepressant agents in adult primary care. Prim Care Companion J Clin Psychiatry 5: $153-157$

Saunders K, Stergachis A, Von Korff M (1994) Group Health Cooperative of Puget Sound. In Pharmacoepidemiology, Strom, B.L. (ed) John Wiley \& Sons: New York, pp 171-185

Sharpe CR, Collet JP, Belzile E, Hanley JA, Boivin JF (2002) The effects of tricyclic antidepressants on breast cancer risk. Br J Cancer 86: $92-97$

Stafford RS, MacDonald EA, Finkelstein SN (2001) National patterns of medication treatment for depression, 1987-2001. Prim Care Companion J Clin Psychiatry 3: $232-235$

Steingart A, Cotterchio M, Kreiger N, Sloan M (2003) Antidepressant medication use and breast cancer risk: a case-control study. Int $J$ Epidemiol 32: $961-966$

Surveillance, Epidemiology and End Results (SEER) (2005) Available at http://www.seer.cancer.gov. National Cancer Institute

Taplin SH, Thompson RS, Schnitzer F, Anderman C, Immanuel V (1990) Revisions in the risk-based Breast Cancer Screening Program at Group Health Cooperative. Cancer 66: $812-818$

Trentham-Dietz A, Newcomb PA, Storer BE, Remington PL (2000) Risk factors for carcinoma in situ of the breast. Cancer Epidemiol Biomarkers Prev 9: 697-703

Tworoger SS, Eliassen AH, Rosner B, Sluss P, Hankinson SE (2004) Plasma prolactin concentrations and risk of postmenopausal breast cancer. Cancer Res 64: 6814-6819

van Schaik N, Graf U (1991) Genotoxicity evaluation of five tricyclic antidepressants in the wing somatic mutation and recombination test in Drosophila melanogaster. Mutat Res 260: 99-104

van Schaik N, Graf U (1993) Structure-activity relationships of tricyclic antidepressants and related compounds in the wing somatic mutation and recombination test of Drosophila melanogaster. Mutat Res 286: $155-163$

Wang PS, Walker AM, Tsuang MT, Orav EJ, Levin R, Avorn J (2001) Antidepressant use and the risk of breast cancer: a non-association. J Clin Epidemiol 54: 728-734

Weiss HA, Brinton LA, Brogan D, Coates RJ, Gammon MD, Malone KE, Schoenberg JB, Swanson CA (1996) Epidemiology of in situ and invasive breast cancer in women aged under 45. Br J Cancer 73: 1298-1305

West SL, Savitz DA, Koch G, Strom BL, Guess HA, Hartzema A (1995) Recall accuracy for prescription medications: self-report compared with database information. Am J Epidemiol 142: 1103-1112 